\title{
Modeling of the thermal contact resistance of a solid-solid contact
}

\author{
Chadouli Rachid, Makhlouf Mohammed \\ Mechanical Engineering DepartmentFaculty of Technology University of Sidi.Bel.Abbes 22000 Algeria, \\ Sidi bel Abbes University, Faculty of Engineering, Algeria
}

\begin{abstract}
At the contact interfaces of solid-solid, we talk about real contact versus a perfect contact when after loading; there is a big difference between the real contact area and the nominal contact area. The objective is usually to obtain perfectly smooth surfaces. The work done in this paper is to study the heat transfer between two solids imperfect contact. For this, we used the numerical simulation by the fluent code release (6.2.16). It should be noted that the determination of the criteria and mechanisms of heat transfer depends strongly on the thermal contact resistance TCR which is produced by the imperfection of the solid-solid contact. The objective of this study was to minimize the TCR to improve the passage of heat flow in the contact interface. To do this, we set up two analytical and numerical solutions to calculate the temperature and the amount of heat flow, to study the influence of the evolution of the contact area due to the progressive loading of the heat flux and the thermal contact resistance.
\end{abstract}

Keys words: Interface, loading, thermal contact resistance $T C R »$, contact surface.

\section{Introduction}

Knowledge of the thermal contact resistance from a model is interesting for the simulation has grown considerably over the past two decades in almost all sectors of the industry ( aerospace, automotive, public works and electronic); the purpose of the simulation is often to make the sizing calculation of a thermal system or studying the improvement of production processes or formatting.

For a long time, the analysis of heat transfer through the solid/solid contact interfaces (the very thin heterogeneous layer) which extends on both sides of the theoretical contact arouses interest and identifies many works dedicated to the characterization of interfaces as the modeling of the thermal contact resistance "TCR". There may be mentionedthe recentworkof Belghali . M [ 1 ], Larzabal.C [ 2 ] and Assefraoui. A [ 3] which have focused on the study of the interface structure. The main objective of this work is to provide or verify theoretical models of thermal contact resistance static " TCR " applicable to various configurations.

In fact we are interested in the evolution of the structure of the interface solid solid contact / between a smooth and infinitely rigid transparent material, and another rough and deformable. This interface is subject to a progressive loading. The contact parameter of interest is the area of real contact.

So our goal is to develop a methodology for the geometric and double thermal characterization of the contact interface. The aim is to establish the laws of evolution of the thermal contact resistance and heat flux depending on the contact surface, for this purpose, three pairs of materials are used: Steel / Steel, Steel/Copper and Steel/Nickel.

We have seen that the thermal contact resistance is the result of two resistors Rs and Rf. However the last depend on the parameters of contact and the nature of the interstitial medium . The study of contact mechanics shows that, for a given state with a given surface couple the evolution of the contact surface is strongly related to the operating conditions, namely the average interface temperature and pressure contact. Increasing the contact pressure has a very clear effect on the heat transfer between solid fused. This effect was studied practically by many authors $[4,5,6,7,8,9,10,11,12,3]$, in the figure ( I.1.1 ), we give an example of the result proposed by MOKRANI and BOUROUGA [ 13 ] .

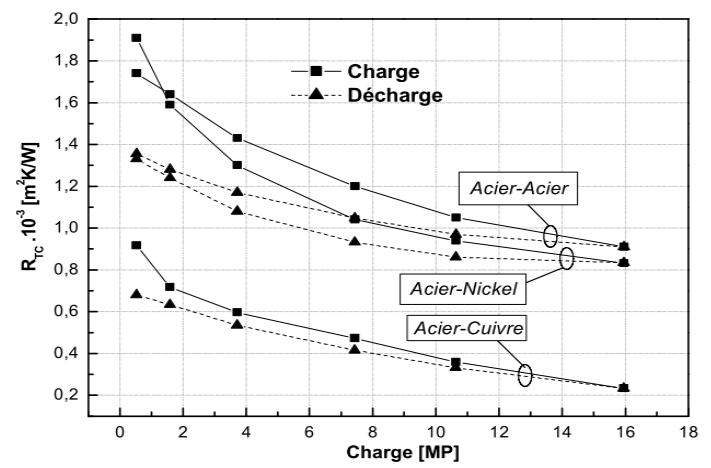

Fig (I.1.1): Variationof the TCR as a functionof the load. [13]. 
The tworesistors $\mathrm{R}_{\mathrm{s}}$ andR $\mathrm{R}_{\mathrm{f}}$ respectively characterizethe passagebythe solidcontact andthetheinterstitial medium. The authors[14, 6, 7, 8, 9, 15, and 16] found thattheapplication of pressureto the contactsectionexpands thesolid-solidcontact, buthas little effect onthe thickness of thefluidinterstitiel.ilsdeducedthatthe contact pressureisalmost exclusively on the $\mathrm{R}_{\mathrm{s}}$ component dueto the phenomenon ofconstriction offlow lines.

\section{I.2. definition of the thermalcontact resistance}

In the multilayer configuration, the quality of the thermal contact between the two layers can bedescribed by a single parameter which is the contact thermal resistance (TCR). In most theoretical studies, it is assumed that the physical contact between two isotropic media is thermally perfect, while in reality, a thermal resistance of significant contact exists because of the presence of a thin intermediate or transition due to irregularities and surface roughness of materials in contact, as well as the possible presence of interstitial phase or impurities which are a barrier to the normal flow of heat flux presence. This resistance is especially important when dealing with solid contact. In this case, two modes of heat transfer are superimposed (Figure I.2.1):

- a transfer by conduction at the contact areas

- $\quad$ a complex transfer through the interstitial fluid.

In the case of the solidconductive medium, there is a convergence of flow lines to the contact areas where heat flow is easier called constriction effect. When the conductivity of the interstitial fluid is similar to that of the medium in contact, the effect of constriction becomes very small and can be neglected. In general, the value of the TCR varies between $10^{-8} \mathrm{~m}^{2} . \mathrm{KW}^{-1}$ (near-perfect contact) and $10^{-4} \mathrm{~m}^{2} . \mathrm{KW}^{-1}$. Different values of the TCR in this range are used to test this model [17], [18].

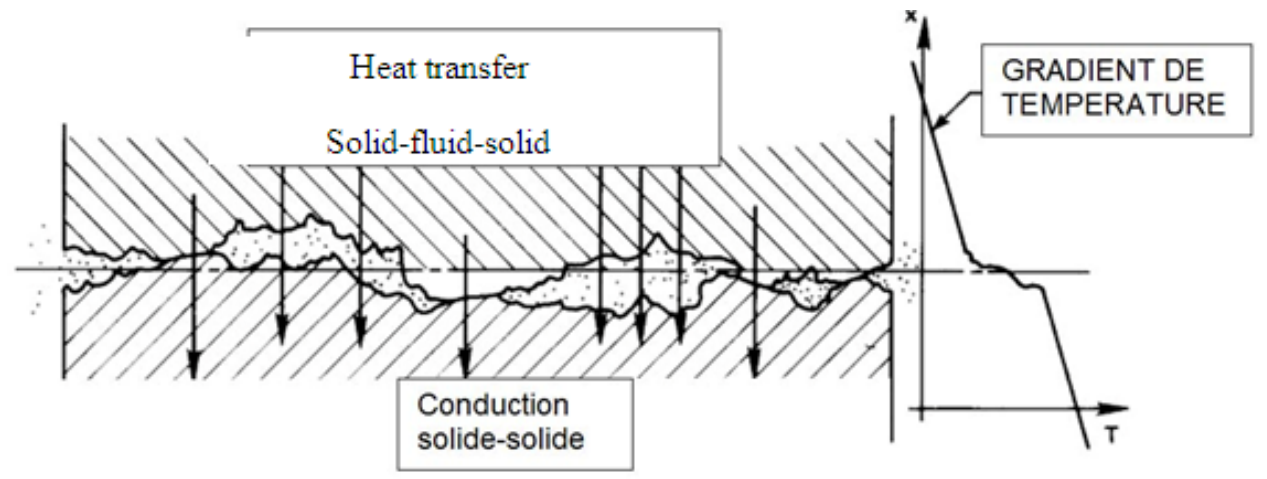

Fig (I.2.1): Heat transferat the interfaceof two solidinimperfect contact[19]

\section{Description of the problem}

When two solids are in contact, due to their roughness and unevenness of the surfaces, the contact is never performed on the entire exposed surface, but only in certain areas of very low surface to the visible surface. Bardon [20] Snaith et al [21] showed that the real contact area is about 1\% of the apparent surface for metals.

Between the contact areas there is an interstitial space generally badconductor, which are a hindrance to heat transfer, which thereby passes preferentially at the contacts where the heat flow is facilitated. The temperature field is therefore significantly disrupted in the localized area either side of the interface. It results a constriction of the flow lines (Fig. II.1) which is responsible for the thermal contact resistance. Bardon [22] . The thermal contact resistance in the steady state is defined by:

$$
\mathrm{TCR}=\frac{\mathrm{T} 2-\mathrm{T} 1}{\varphi}
$$

WhereT1andT2are the twocontact temperaturesextrapolatedfieldundisturbedtemperaturestowards the geometriccontact interface(Fig. I.1.b).

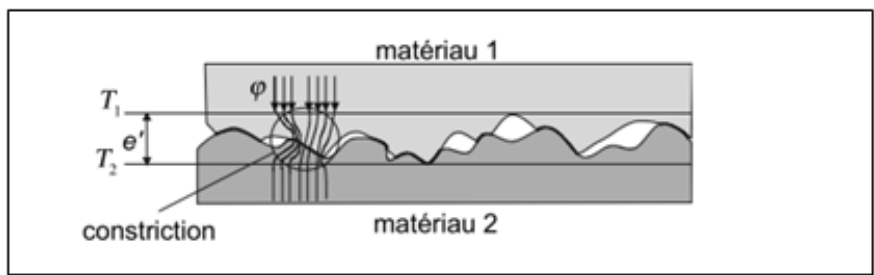

Fig (II.1): Constrictionof the flux linesat thecontact zone. 
To determine the TCR at the interface solid/solid during forming processes, several studies have so far favors an experimental approach to the problem. Analytical and numerical approaches when to allow it to determine, based on models of the TCR values corresponding to the geometric interface conditions and characteristics of materials used.

The mechanical compression loading of an interface solid- solid contact gives rise to a complex field of discrete deformation due to the distribution of contact points. Indeed, the two contacting surfaces will give rise to a quasi- isostatic contact and loading will first multiply the points of contact, increasing the high load will result in the spread of contact points a phenomenon coalescence of these points so the real area of contact changes.

The mechanical evolution of a metal surface is characterized by its topography that is essentially described by a function of the distribution of the heights about the distribution of heights of the rough surface relative to the reference plane, and the roughness parameters which describe the height of the asperities of a rough surface.

Many thermal problems lead us to consider the contact between two solids, one smooth and the other rough and rigid. It is two -dimensional solid plates of length $\mathrm{L}$ and height $\mathrm{H}$, thickness $\delta$ touch filled with fluid, which is in our case air, active walls of the two solids are maintained at two different temperatures and uniform named respectively $T_{C}$ and $T_{f}\left(T_{C}>T_{f}\right)$. Inactive walls are vertical walls $X=0$ and $X=L$, which are thermally insulated. Considering that the contact between the two is imperfect solids applying pressure (FigureII.2)

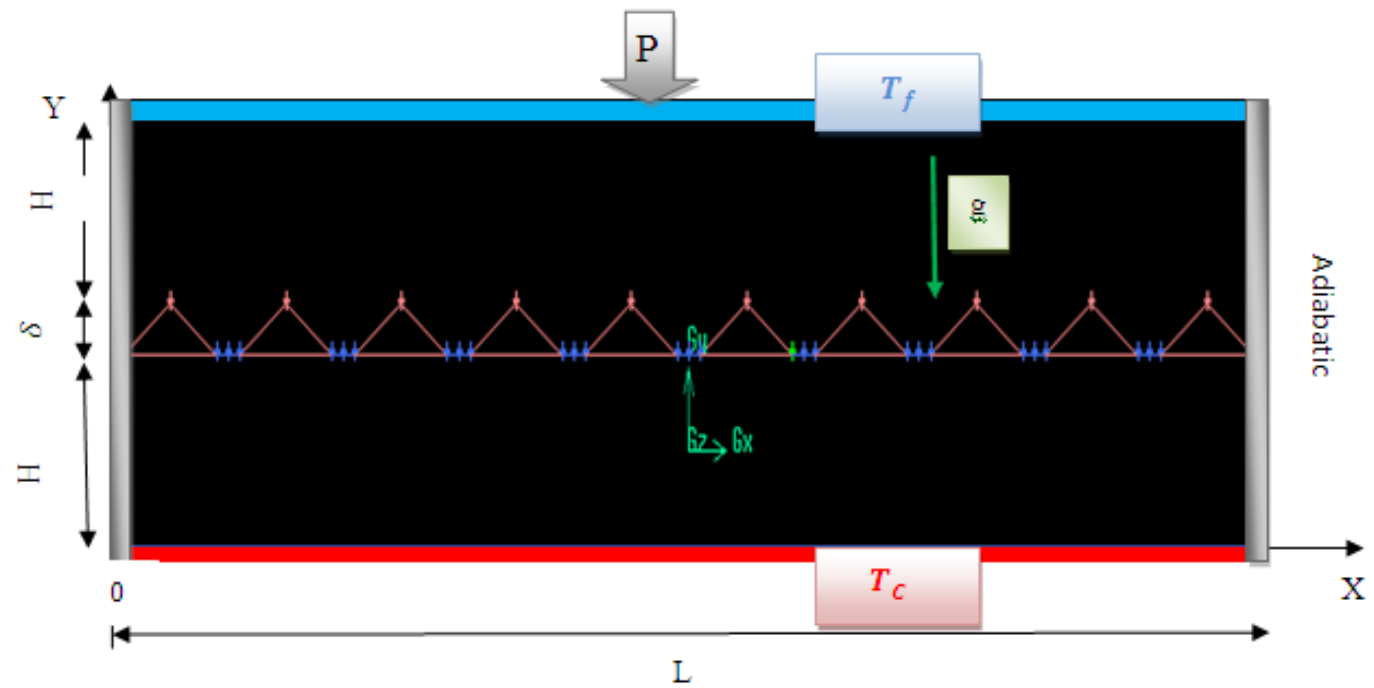

Fig (II.2): Descriptionof the problem

\section{II.2. simplifying assumptions}

In order tosolvethis system of equationsabove,certainassumptions are used:

-Continuousand isotropicmedium.

-Calculation modelis two-dimensionaland stationary.

-Theairis consideredincompressiblefluid and thetransfer modebyradiation negligible.

-Thevolume forcesare only due tothe accelerationof gravity.

-The physical properties ofair areindependent of temperatureexceptthe densityin the equationsof momentum.

-The speedsinvolved areweak andthe internalheat generationis negligibleq $=0$.

-Theflow ofviscousheat dissipationis negligible: $\varphi=0$.

- The term $\beta \mathrm{T} \frac{\mathrm{dP}}{\mathrm{dt}}$ (heating bycompressionpower) is negligible due to the lowspeedsfaceoffs.

-The fluid iscompletely transparent.It does notinvolve in the radiative exchanges.(Noradiation exchangewithin the fluid).

\section{II.3. Equationsgoverningthe problem}

Thecontinuity equationexpressingthe law of conservationof mass foracontrolvolume of materialand the

$$
\frac{\partial \rho}{\partial t}+\operatorname{div}(\rho \vec{V})=0
$$
equationof momentumobtained fromthe secondlaw of dynamicsare respectivelyas follows: 


$$
\frac{D}{D t}(\rho \cdot \vec{V})=\rho \cdot \vec{F}-\operatorname{gra} d(p)+\mu \cdot \Delta(\vec{V})+\frac{1}{3} \cdot \mu \cdot g r \vec{a} d(\operatorname{div}(\vec{V}))
$$

Theconservationof energy equationexpressing thevariationoftotal poweris the sum oftheenergy variationdue to conductionand the internalheat production"q", and powervariation dueto the effect ofcompressibilityandviscousenergydissipation. either:

$$
\frac{D}{D t}\left(\rho \cdot c_{p} \cdot T\right)=\Delta(\lambda \cdot T)+q+\beta \cdot T \cdot \frac{D p}{D t}+\mu \cdot \phi
$$

\section{II.3.1.dimensionalequations}

* Introducingtheabove-mentionedassumptions, we arrive at thefollowingequationsystem:

* Continuity equation:

$$
\frac{\partial u}{\partial x}+\frac{\partial v}{\partial y}=0
$$

\section{* Equationsof momentum:}

Along the axisx :

$$
u \frac{\partial u}{\partial x}+v \frac{\partial u}{\partial y}=-\frac{1}{\rho_{0}} \frac{\partial p}{\partial x}+v\left(\frac{\partial^{2} u}{\partial x^{2}}+\frac{\partial^{2} u}{\partial y^{2}}\right)
$$

Along the axisy :

$$
u \frac{\partial v}{\partial x}+v \frac{\partial v}{\partial y}=-\frac{1}{\rho} \frac{\partial p}{\partial y}+v\left(\frac{\partial^{2} v}{\partial x^{2}}+\frac{\partial^{2} v}{\partial y^{2}}\right)+\beta g\left(T-T_{0}\right)
$$

\section{* EnergyEquation:}

$$
u \frac{\partial T}{\partial x}+v \frac{\partial T}{\partial y}=\alpha\left(\frac{\partial^{2} T}{\partial x^{2}}+\frac{\partial^{2} T}{\partial y^{2}}\right)
$$

Where:' $v$ ' is the kinematicviscosity and, $\alpha=\frac{\lambda}{\rho c_{p}}$ the thermal diffusivityof the fluid.

These differentialequationswith partial derivatives form themathematical model of theflowlaminarnatural convectionof our problem.

\section{II.3.2.dimensionlessequations}

Theadimensionnalisationornormalizationis to transformthe dependent and independentvariablesindimensionless variables, that is to say, theywill benormalized with respectto certain characteristicsdimensions. Thisallows you to specifythe flow conditionswith alimited number of parametersto make theoverall solution.

In the processes ofheat transferby natural convection,the formulation indimensionlessvariablesis importantto simplifythe equationsgoverningthe flowandto guideexperimentsto be performed.

In order to makethe above equationsin dimensionlessform, the following variablesare introduced:

\section{* Continuity equation:}

$$
\mathrm{Y}=\frac{\mathrm{y}}{\mathrm{H}}, \mathrm{X}=\frac{\mathrm{x}}{\mathrm{L}}, \mathrm{U}=\frac{\mathrm{u}}{\mathrm{vH} / \mathrm{L}^{2}}, V=\frac{\mathrm{v}}{\mathrm{v} / \mathrm{L}}, \theta=\frac{\mathrm{T}-\mathrm{T}_{\mathrm{c}}}{\mathrm{T}_{\mathrm{f}}-\mathrm{T}_{\mathrm{c}}}, \mathrm{P}=\frac{\mathrm{p}}{\rho_{0}(\mathrm{v} / \mathrm{L})^{2}}
$$

$$
\frac{\partial U}{\partial X}+\frac{\partial V}{\partial Y}=0
$$

\section{* Equationsof momentum:}


Along the axis $x$ :

$$
U \frac{\partial U}{\partial X}+V \frac{\partial U}{\partial Y}=-\frac{\partial P}{\partial X}+\frac{1}{A r^{2}} \frac{\partial^{2} U}{\partial X^{2}}+\frac{\partial^{2} U}{\partial Y^{2}}
$$

Along the axis $\mathbf{y}$ :

$$
U \frac{\partial V}{\partial X}+V \frac{\partial V}{\partial Y}=-\frac{1}{A r^{2}} \frac{\partial P}{\partial Y}-\frac{G r}{A r} \theta+\frac{1}{A r^{2}} \frac{\partial^{2} V}{\partial X^{2}}+\frac{\partial^{2} V}{\partial Y^{2}}
$$

\section{* EnergyEquation:}

$$
U \frac{\partial \theta}{\partial X}+V \frac{\partial \theta}{\partial Y}=\frac{1}{P r}\left[\frac{1}{A r^{2}} \frac{\partial^{2} \theta}{\partial X^{2}}+\frac{\partial^{2} \theta}{\partial Y^{2}}\right]
$$

Therefore, theconservation equationsadimensionnalisationyieldedthedimensionless numbers, which characterizefluid flowandheat transferbetween two solidcontacting.

\section{$>$ Number ofGrashof}

It is adimensionless numberusedin fluid mechanicsto characterize thenatural convectionin a fluid.Itis the ratioof gravitational forcestoviscous forces. It is definedby:

$$
G r=\frac{g \beta \Delta T L_{c}{ }^{3}}{v^{2}}
$$

Where $\mathrm{L}_{\mathrm{C}}$ :The characteristic lengthbetween the hotandcold wall.

\section{Number ofRayleigh}

It is adimensionless number, astheheat transfercharacteristicwithin afluid.This number is usedin fluid mechanics. Belowa critical value of 2000 , thetransfer takes placeby conduction, beyondthis value, it is the freeconvectionbecomes important.It is definedas follows:

$$
R a=\frac{g \beta \Delta T L_{c}{ }^{3}}{v \alpha}=G r \cdot P r
$$

\section{$>$ Number ofPrandtl :}

It is adimensionlessnumber.It representsthe ratio of thediffusivityof momentum(orkinematic viscosity) and thermal diffusivity. It is definedas follows:

$$
\operatorname{Pr}=\frac{v}{\alpha}
$$

\section{II.4.Boundary conditions}

Solvingthe system of equationsobtained aboverequiresthe incorporation ofboundary conditions foreach variable. The temperature conditionsare knownon the walls.

Theconditionsassociated with the problemlimits are:

$\mathrm{t}>0: \mathrm{u}=\mathrm{v}=0, \mathrm{y}=0$, on the hot wall, $\mathrm{T}=\mathrm{T}_{\mathrm{C}} . \mathrm{y}=\mathrm{L}$, on the cold wall, $\mathrm{T}=\mathrm{T}_{\mathrm{f}}$.

- $\quad$ Terms ofadiabaticx $=0$ et $H: \frac{\partial \theta}{\theta x}=0 . \quad y=0$ et $L: \frac{\partial \theta}{\theta y}=0$.

\section{- Boundary conditionsin dimensionlessform}

$\tau>0: \mathrm{U}=\mathrm{V}=0, \quad \frac{\partial \theta}{\theta \mathrm{Y}}=0 \quad$ à $\mathrm{Y}=0$ et 1 ,

$\theta=1$, on the hot wallà $X=0$,

$\theta=0$, on the cold wall à $X=1$, 


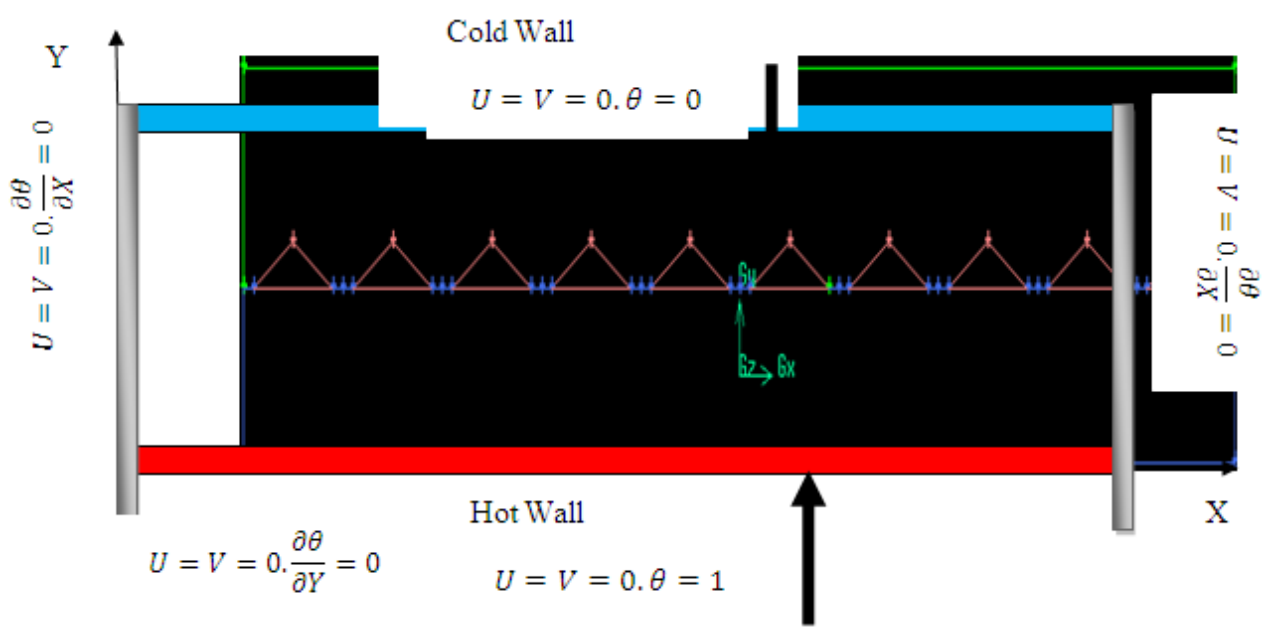

FIG ; (II.4.1):Boundary conditions.

\section{Descriptionof the objectives ofour study}

The thermal contact resistance at the interface reflects the heat flow in parallel through both direct solid-solid track where the flow must pass through a constriction resistance denoted by $\mathrm{R}_{\mathrm{S}}$ and indirect path through the nip characterized by resistance of the fluid blade denoted $R_{\mathrm{f}}$. It is therefore considered as the resultant of these two parallel resistors $\mathrm{R}_{\mathrm{s}}$ and $\mathrm{R}_{\mathrm{f}} \mathrm{as}$ :

$\frac{1}{\mathrm{RTC}}=\frac{1}{\mathrm{Rs}}+\frac{1}{\mathrm{Rf}}$

The contact areas between an interstitial space remains generally poor conductor, which is a barrier to heat transfer, which thereby passes preferentially at the contacts where the heat flow is facilitated. The temperature field is thereby significantly disturbed in the localized region of each side of the interface. This results in a constriction of the flow lines is responsible for the thermal contact resistance (RTC) .

In this work we are interested in the numerical study of the thermal conduction behavior at the points of contact and by natural convection air flow in the interstitial space between the two horizontal solid contact in $2 \mathrm{D}$, length $\mathrm{L}=2 \mathrm{~mm}$, and $0.15 \mathrm{~mm} \mathrm{D}=$ width of thickness $\delta$, considering a rigid smooth surface and the other a rough triangular asperities of the same size. And the distribution of the mesh points and numerical simulation were made respectively in a mesh Gambit and Fluent CFD code.

The study is based on the influence of the pressure way which is through the development of the contact surface of the contact thermal resistance on the one hand, and on the other hand the heat flux . To this end we made five attempts on three pairs of solid materials ( steel-steel, steel - copper andsteel -nickel) .

\section{Numerical Simulation}

Various problems of fluid mechanics are governed by the same equations, only the boundary conditions can be distinguished. The following boundary conditions are defined by the FLUENT code. At the entrance of the wall 4 to a temperature $\mathrm{T}=500 \mathrm{~K}$ and the wall 1 has a temperature $\mathrm{T}=300 \mathrm{~K}$ with $\mathrm{g}=9.81 \mathrm{~m} / \mathrm{s}^{2}$.

\section{IV.1.1.Initial conditions}

We initializethe parameters for calculatingrelative toconditions chosenlimits. The fluid used inthis study is theairfollowing physical properties:
$\rho=1 \mathrm{~kg} / \mathrm{m}^{3}$
$\mathrm{Cp}=4.185 \mathrm{j} / \mathrm{kg} \cdot \mathrm{k} \mu=0.048 \mathrm{~kg} / \mathrm{m} . \mathrm{s}$
$\lambda=0.12 \mathrm{w} / \mathrm{m} \cdot \mathrm{k}$

\section{V.1.2. measurementprinciple:}

To perform the calculation, we have imposed a temperature $T_{1}=500 \mathrm{k}$ on the wall of smooth and rigid solid (wall 4 is the hot wall) and the wall of rough solid temperature $\mathrm{T}_{2}=300 \mathrm{~K}$ (one wall is the cold wall). We did the math for three couples and generated for each case studied the influence of temperature in the transition zone with the fluent code for a calculation of temperature at the interstitial interface.

\section{IV.1.3. Les contours of temperature Static:}

To determine the degradation temperature we define the line temperature in the region of contact.fig (V.1) of the torque material for Example Steel / Copper. 


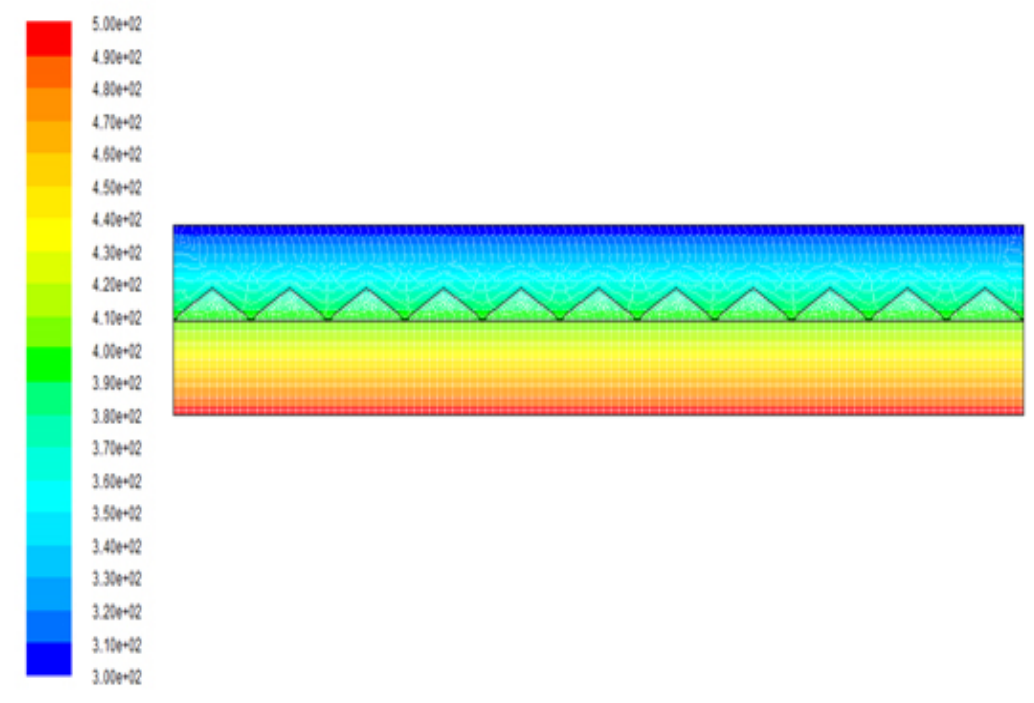

FIG ; (IV.1): Fielddegradationtemperaturein solidcontact with $\delta=0.05 \mathrm{~mm}$

The variation ofthe static temperaturegradientincreases withthe evolution ofroughsurfaces.

\section{IV.2.Contourtemperature variation}

Fordetermining the variationof the temperature inthe transition regionthere are twolinesin the areabetween the two solidas shownin Figure(IV.2) fortorqueSteel /Copper.

MeasuringtheRTCbetween twosolids instatic contactis to createperpendicular tothe contact surface, a unidirectional flowofheat in the areanotaffected bytheconstrictionofflux lines. This flowand thetemperature jumpat the interface aredetermined from themeasured temperaturesonthe contact area. The followingtablesrepresent the measurementsand calculations offlow andTCRfor three couplesmaterials.

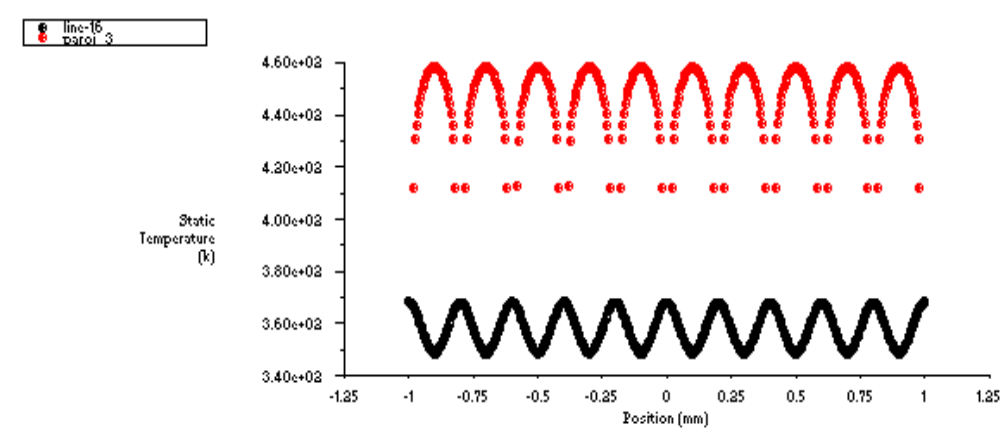

\begin{tabular}{|c|c|}
\hline Ststic Tempersture & 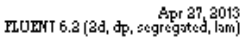 \\
\hline
\end{tabular}

FIG ;(IV.2) :temperature variation betweenthetworows in thecontact zone.

\begin{tabular}{|c|c|c|c|c|c|}
\hline fusedsolid & & & steel/steel & & \\
\hline tests & 1 & 2 & 3 & 4 & 5 \\
\hline solid surface $\left(\mathrm{mm}^{2}\right)$ & 0.05 & 0.2 & 0.4 & 0.6 & 0.8 \\
\hline$\lambda_{s}(\mathrm{w} / \mathrm{m} . \mathrm{K})$ & 16.27 & 16.27 & 16.27 & 16.27 & 16.27 \\
\hline$\lambda_{\mathrm{f}}(\mathrm{w} / \mathrm{m} . \mathrm{K})$ & 0.0242 & 0.0242 & 0.0242 & 0.0242 & 0.0242 \\
\hline$\Delta \mathrm{T}(\mathrm{K})$ & 198.9 & 109.53 & 90.65 & 68.89 & 45.00 \\
\hline $\mathrm{TCR}_{\mathrm{s}}(\mathrm{K} / \mathrm{W})$ & 0.06146281 & 0.01229256 & 0.00507068 & 0.00256095 & 0.00145974 \\
\hline $\mathrm{TCR}_{\mathrm{T}}(\mathrm{K} / \mathrm{W})$ & 0.06145367 & 0.01229227 & 0.00507064 & 0.00256094 & 0.00145974 \\
\hline Flux $_{f}(\mathrm{~W})$ & 0.481338 & 0.21205008 & 0.1316238 & 0.06668552 & 0.02178 \\
\hline Flux $_{\mathrm{s}}(\mathrm{W})$ & 3236.1030 & 8910.2655 & 17877.2788 & 26900.1672 & 30827.3684 \\
\hline Flux $_{\mathrm{T}}(\mathrm{W})$ & 3236.58434 & 8910.47755 & 17877.4104 & 26900.2339 & 30827.3902 \\
\hline
\end{tabular}


TableIV.2.1 :calculation of the parametersoftorqueSteel /Nickel

\begin{tabular}{|c|c|c|c|c|c|}
\hline fusedsolid & & & steel/ Copper & & \\
\hline tests & 1 & 2 & 3 & 4 & 5 \\
\hline thickness (mm) & 0,05 & 0,04 & 0,033 & 0,025 & 0,019 \\
\hline solid surface $\left(\mathrm{mm}^{2}\right)$ & 0,05 & 0,2 & 0,4 & 0,6 & 0,8 \\
\hline fluid Surface $\left(\mathrm{mm}^{2}\right)$ & 0,005 & 0,0032 & 0,00198 & 0,001 & 0,00038 \\
\hline$\lambda_{\mathrm{s}}(\mathrm{w} / \mathrm{m} . \mathrm{K})$ & 387,6 & 387,6 & 387,6 & 387,6 & 387,6 \\
\hline$\lambda_{\mathrm{f}}(\mathrm{w} / \mathrm{m} . \mathrm{K})$ & 0,0242 & 0,0242 & 0,0242 & 0,0242 & 0,0242 \\
\hline$\Delta \mathrm{T}(\mathrm{K})$ & 196,23 & 106,15 & 80,56 & 66,67 & 39,21 \\
\hline $\mathrm{TCR}_{\mathrm{f}}(\mathrm{K} / \mathrm{W})$ & 413,22314 & 516,528926 & 688,705234 & 1033,05785 & 2066,1157 \\
\hline $\mathrm{TCR}_{\mathrm{s}}(\mathrm{K} / \mathrm{W})$ & 0,00257998 & 0,000516 & 0,00021285 & 0,0001075 & $6,1275 \mathrm{E}-05$ \\
\hline $\mathrm{TCR}_{\mathrm{T}}(\mathrm{K} / \mathrm{W})$ & 0,00257996 & 0,000516 & 0,00021285 & 0,0001075 & $6,1275 \mathrm{E}-05$ \\
\hline $\operatorname{Flux}_{\mathrm{f}}(\mathrm{W})$ & 0,4748766 & 0,2055064 & 0,11697312 & 0,06453656 & 0,01897764 \\
\hline Flux $_{\mathrm{s}}(\mathrm{W})$ & 76058,748 & 205718,7 & 378485,527 & 620191,008 & 639907,2 \\
\hline $\operatorname{Flux}_{\mathrm{T}}(\mathrm{W})$ & 76059,2229 & 205718,906 & 378485,644 & 620191,073 & 639907,219 \\
\hline
\end{tabular}

TableIV.2.2 :Calculation of the parametersoftorquesteel-Copper .

\begin{tabular}{|l|c|c|c|c|c|}
\hline \multicolumn{1}{|c|}{ fusedsolid } & \multicolumn{3}{c|}{ Steel /Nickel } \\
\hline tests & 1 & 2 & 3 & 4 & 5 \\
\hline thickness $(\mathrm{mm})$ & 0,05 & 0,04 & 0,033 & 0,025 & 0,019 \\
\hline solid surface $\left(\mathrm{mm}^{2}\right)$ & 0,05 & 0,2 & 0,4 & 0,6 & 0,8 \\
\hline fluid Surface $\left(\mathrm{mm}^{2}\right)$ & 0,005 & 0,0032 & 0,00198 & 0,001 & 0,0038 \\
\hline $\boldsymbol{\lambda}_{\mathbf{s}}(\mathrm{w} / \mathrm{m} . \mathrm{K})$ & 91,74 & 91,74 & 91,74 & 91,74 & 91,74 \\
\hline $\boldsymbol{\lambda}_{\mathbf{f}}(\mathrm{w} / \mathrm{m} \cdot \mathrm{K})$ & 0,0242 & 0,0242 & 0,0242 & 0,0242 & 0,0242 \\
\hline$\Delta \mathrm{T}(\mathrm{K})$ & 199,03 & 106,87 & 83,06 & 57,01 & 40,58 \\
\hline $\mathrm{TCR}_{\mathrm{f}}(\mathrm{K} / \mathrm{W})$ & 413,22314 & 516,528926 & 688,705234 & 1033,05785 & 206,61157 \\
\hline $\mathrm{TCR}_{\mathrm{s}}(\mathrm{K} / \mathrm{W})$ & 0,01090037 & 0,00218007 & 0,00089928 & 0,00045418 & 0,00025888 \\
\hline $\mathrm{TCR}_{\mathrm{T}}(\mathrm{K} / \mathrm{W})$ & 0,01090008 & 0,00218006 & 0,00089928 & 0,00045418 & 0,00025888 \\
\hline Flux $_{\mathrm{f}}(\mathrm{W})$ & 0,4816526 & 0,20690032 & 0,12060312 & 0,05518568 & 0,1964072 \\
\hline Flux $_{\mathrm{s}}(\mathrm{W})$ & 18259,0122 & 49021,269 & 92362,72 & 125522,338 & 156749,861 \\
\hline Flux $_{\mathrm{T}}(\mathrm{W})$ & 18259,4939 & 49021,4759 & 92362,8406 & 125522,393 & 156750,057 \\
\hline
\end{tabular}

TableIV.2.3:Calculation of the parametersoftorquesteel-Nickel.

\section{Results and discussion}

To determine thethermalcontact resistanceandheat fluxat the interface, we present the results of thetables onthe graphsof the influence oftemperature and the realcontact areaattheTCRand the flowfor eachpair of materialsandthe comparison.

V.1.1. Case of Steel- steel contact

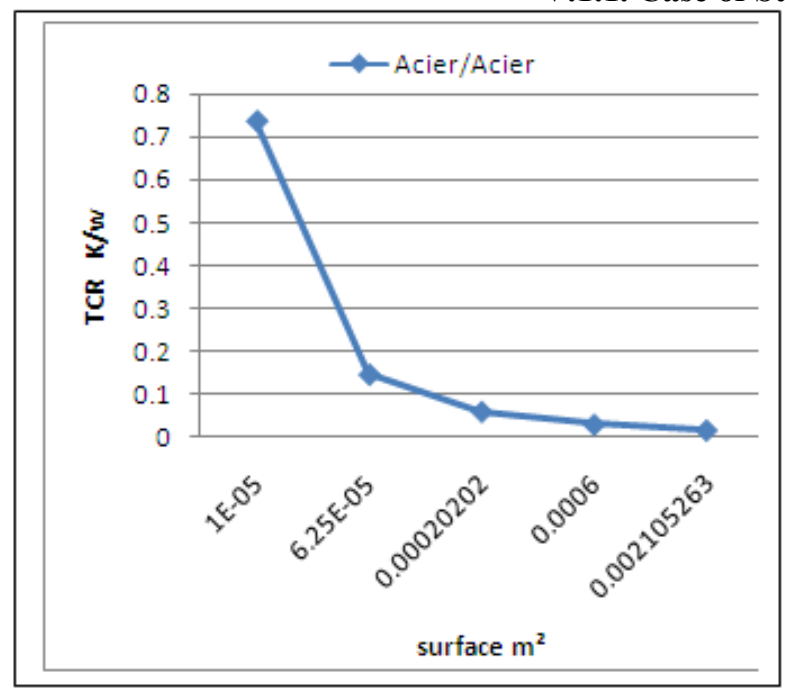

Fig :V.1: variationof the TCRas a function ofthe contact area

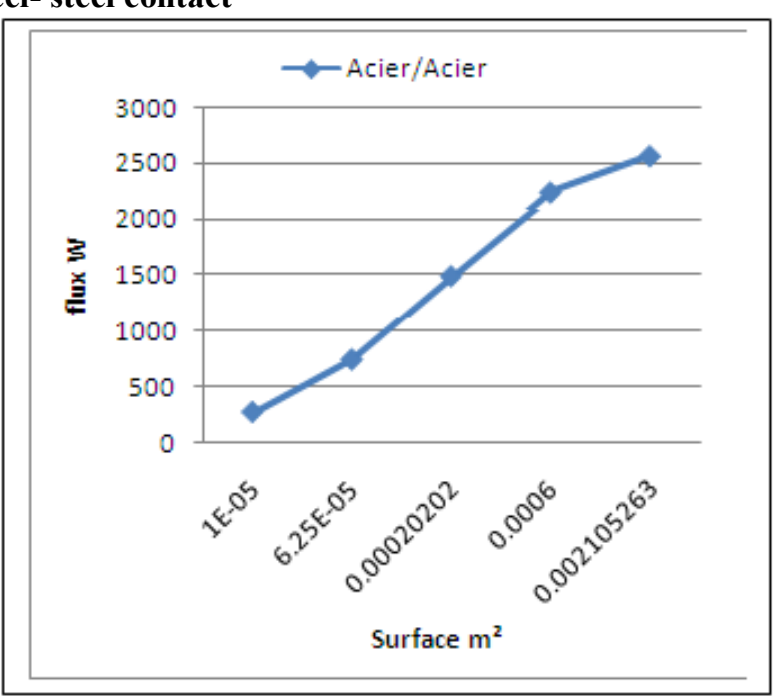

Fig :V.2: variationof the heat flowas a function ofthe contact area 


\section{V.1.2. Case of Steel- Copper contact}

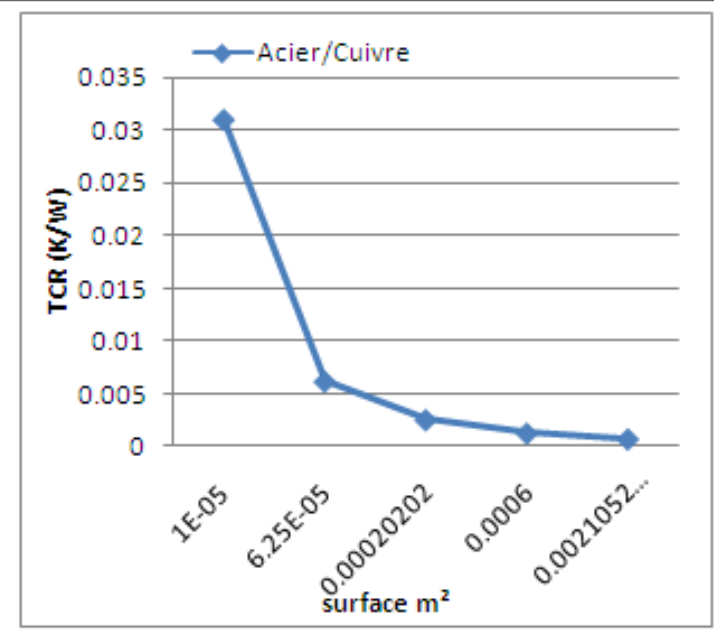

Fig :V.3: variationof the TCRas a function ofthe contact area

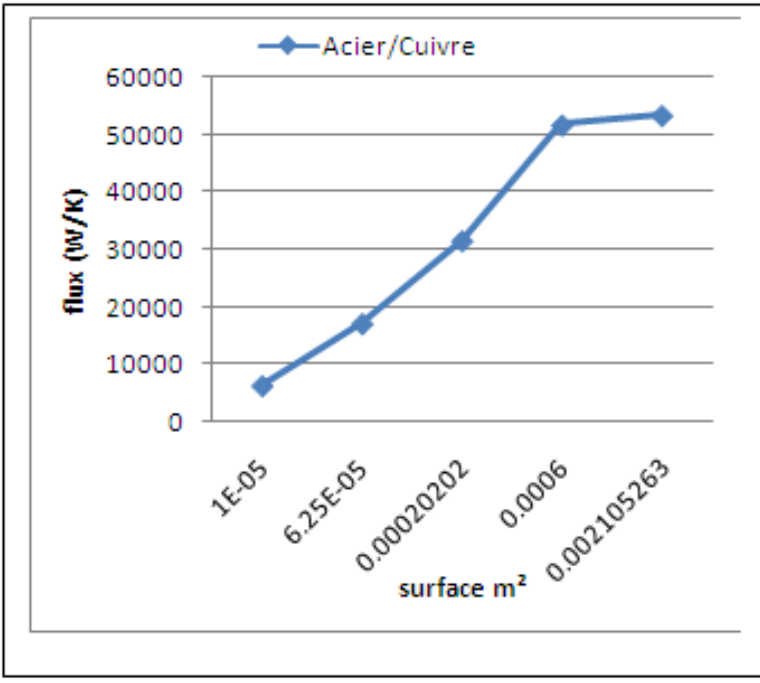

Fig :V.4: variationof the heat flowas a function ofthe contact area

\section{V.1.3.Case of Steel- Nickel contact}

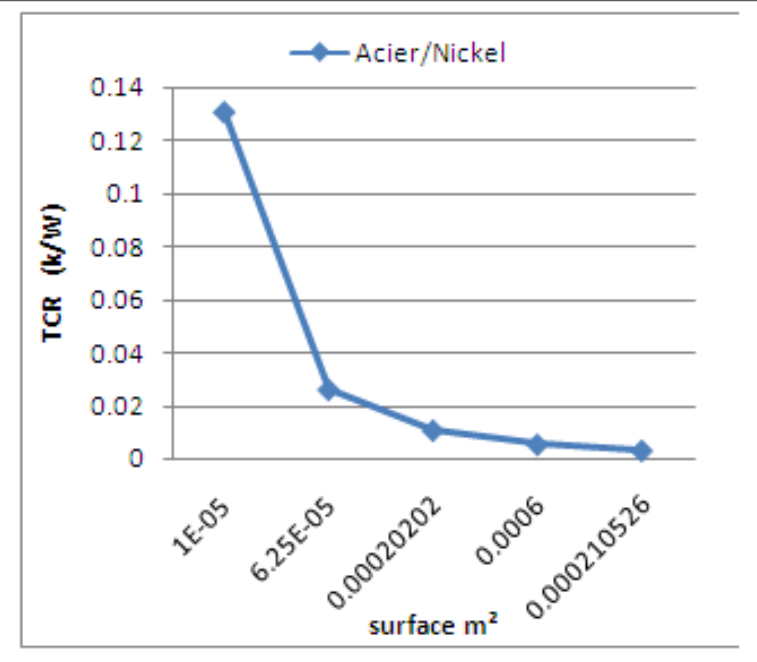

Fig :V.5: variationof the TCR as a function ofthe contact areaofthe contact area

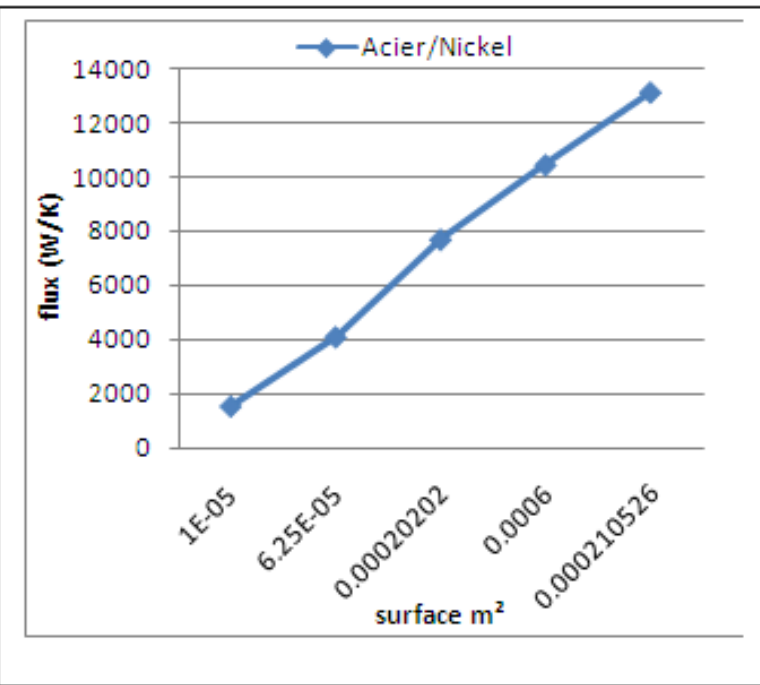

Fig :V.6: variationof the heat flowas a function

Figures(V.1), (V.3) and(V.5) represent the resistance curvebased onthe actualcontact surface forthreepairs of materials. Fromthese graphs, we see that theRTCdecreases with progressive loadingthat we see by the actual contact area.

Figures(V2), (V4) and(V6) representthe curveof heat flowas a function ofthe actualcontact surface forthreepairs of materials. Through thesecurves, we note thattheincrease in the realcontact areadue to theprogressive loadingimprovesthe passage ofheat flow. 
V.2. Comparison of resultsfor the threepairs of materials.

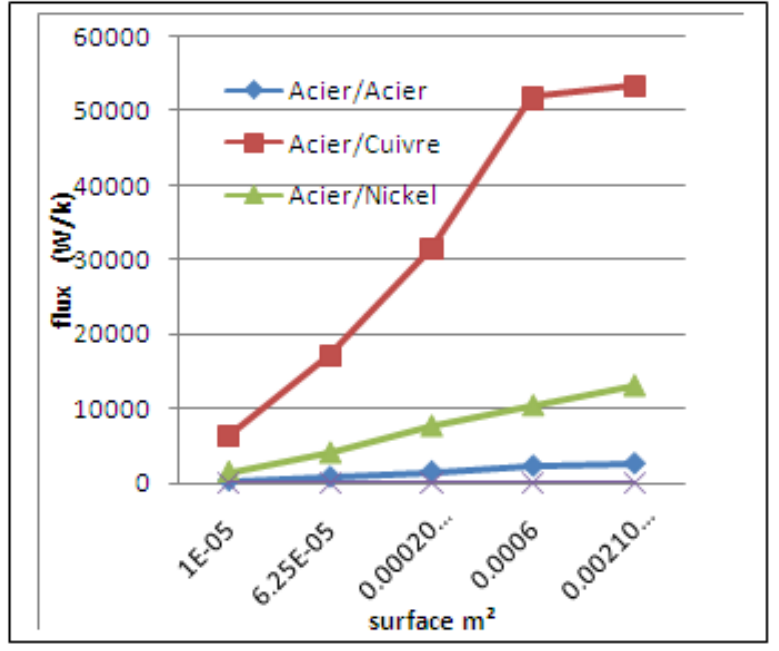

Fig V.7: variationof the heat flowas a function ofthe contact area ofthe contact area

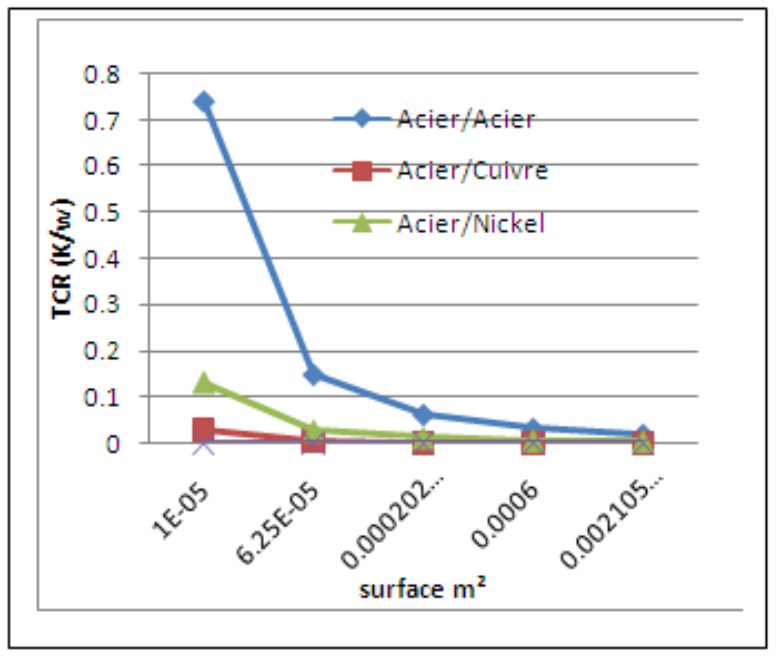

Fig V.8: variationof the TCRas a function

Comparing the values of the TCR for three couples, we see that for the couple-Steel Copper the value of the TCR tends to a minimum value at the end of the charging cycle. (Figure V.8).

For values of the heat flux, we note that the heat flux transmitted through the couple Steel/Copper reaching very satisfactory values for progressive loading. (Fig V.7).

\section{Conclusion}

The objective of this work was to contribute to the understanding of when thermal phenomena including the generation of heat by conduction in the imperfect contacts and influence of the flow of heat for this, we conducted an analysis thermal couples of steel / steel, steel / copper and steel / nickel in planar contact with passage of the temperature field. In the thermal modeling of an imperfect contact, we took into account the influence of some parameters on the evolution of the thermal contact resistance at the interfacial contact, which are: the load, and the contact surface temperature. The torque of the material is one of the most influencing parameters on the thermal behavior. It was found that the torque Steel / Copper we chose supports more heat than the other couple steel / steel or nickel in the heterogeneous area. The comparison between the results of these three materials showed that the heat flux at the contact steel / copper transmitted better than others, because of the low thermal contact resistance. The increase in the latter leads to a decrease in heat flow.

The results show that the most influencing parameters on the thermal contact resistance are: load, type of material, the surface of contact. Therefore, to increase the heat transfer at the contact surface, can be played on these settings to limit or minimize disruption of the flow of heat between the two contiguous solid. The first simulation results show that the evolution of the TCR depending on the contact pressure (contact surface ) is coherent and consistent with the literature. Simulation results show that the couple Steel / Copper is the best conductor of heat transfer ( heat flux ) in the heterogeneous area because of the high conductivity compared to other couples steel / nickel and steel / steel which is the couple lower. The parameters that influence the thermal contact resistance (TCR ) are progressive load ( changing the actual contact surface ) as can be seen in the results in Figure V.8, the interstitial fluid ( as roughness ) and the type of material (thermal conductivity ). The comparison of our simulation results on the thermal contact resistance as a function of pressure is way through contact surface shown in Figure ( V.8 ) with the experimental results shown in MOKRANI.BOUROUGA Figure ( I.1.1) shows that the two results are consistent with an error rate that is justified by the conditions, assumptions configuration and description of the geometry of the problem.

\section{Références}

[1]. M.Belghali- Adil « étude de l'effet de la distribution des aspérités de surface sur la résistance thermique de contact métalliques pressées ».Thèse de doctorat, école polytechnique de l'université de Nantes, 1995.

[2]. C. Larzabal. " approche fractales des effets de la rugosité dans le contact thermique des surfaces métalliques accolés ». Thèse de doctorat, école polytechnique de l'université de Nantes, 2000.

[3]. A. Assefraoui. «Étude optique, mécanique et thermique simultanée et sous pression d'écrasement, de la résistance thermique et des microdéformations Aluminium-Saphir. Comparaison avec un modèle prédictif ». Thèse de doctorat, école polytechnique de l'université de Nantes, 1995.

[4]. Mc Waid T. et Marschal E. «A comparison of elastic and plastic contact models for the prediction of thermal contact conductance". Warme und Stoffubertragung, 28, p: 441-448; 1993.

[5]. Cordier.H « étude expérimentale des résistances thermique de contact. Influence de la pression » Anales de physique, Fév.1961. 
[6]. Maimi.R « contribution à l'étude de l'influence de la pression sur les résistances de contact » Thèse docteur ingénieur, Poitier 1962.

[7]. Roiron G. " contribution à l'étude de l'expérimentale et théorique des résistances thermiques de contact ». Thèse Docteur Ingénieur, Poitiers 1964.

[8]. Fouche F. « étude de l'expérimentale et théorique de la résistance thermique d'un contact par bandes parallèles ». Thèse Doctorat d'état, Poitiers 1966.

[9]. Bardon J.P. « contribution à l'étude du transfert de la chaleur au contact de deux matériaux ». Thèse de Doctorat 1965. La faculté des sciences de l'université de Poitiers.

[10]. Bardon J.P., Cassagne B, Fourcher B, et St Blanquet C. « Bilan des principales recherches sur les résistances thermique de contact ». Rapport D.E.T.B. 7101. Université de Nantes, Mars 1971.

[11]. Manach G. "étude expérimentale et théorique des résistances thermiques de contacts à hautes températures». Thèse Docteur Ingénieur, Poitiers 1966

[12]. F. Dominique, « Essais mécaniques des Métaux » M4 160 Technique d'ingénieur.

[13]. Mokrani. Bourouga: Mesure des paramètres de contact à une interface de contact électrothermique imparfaitLaboratoire de Thermocinétique, UMR CNRS 6607, E.P.U.N., rue Christian Pauc, La Chantrerie, B.P. 90604, 44306 NANTES, Cedex 03, France.

[14]. Cetinkale et fishenden. « Internationalconference on heat transfer » Institution of Mechanical Engineers, London, 1951.

[15]. Holm. « Electric contact » Hogo Gerbers Frlags. Stockholm, 1949.

[16]. Weells N.D et Rryder .E.A. "Thermal résistance measurement of joints formed between stationary metal surface" Trans.Am. Soc. Mech. Engrs 71, 259, 1949.

[17]. Van Schel. Heuret et Bissieux : modélisation, expérimentation et application Thermal contact résistance between metallic materials: measurement by photothermal radiometry. Modelling, expérimentation and application.Revue générale de thermique 1992, 31, $\mathrm{P}$ 291292.

[18]. Salgon. Quemener et Belghali: Résistance thermique de contact statique. Evaluation expérimentale des performances d'un modèle à deux résistances issu d'une description probabiliste. Revue générale de thermique 1998, 37, $284-294$

[19]. Jean-Luc Battaglia, Andrzej Kusiak, Jean-Rodolphe Puiggali. Introduction Aux Transferts Thermique.CFC, 20,rue des GrandsAugustins ,75006 Paris Dunod, Paris, 2010.

[20]. Bardon,j.p. introction à l'étude des résistances thermiques de contact. Revue générale de thermique. Mai $1972 \mathrm{n}^{\circ} 125$, PP $429-446$

[21]. B.Snaith et al. Thermal résistance of pressed contacts. Applied energy ,1986, vol 22, PP 34-84.

[22]. Bardon,j.p. Bilan des principales recherche sur les résistances thermique de contact, Rapport D.E.T.P. 7101, entropie, Mard 1971, $n^{\circ} 64$, PP $1-34$. 